\title{
Solid-State Nonlinear Optical Switch with the Widest Switching Temperature Range Owing to its Continuously Tunable $T_{c}$
}

Chun-Ya Pan,${ }^{\dagger}$ Xin-Rui Yang,${ }^{\dagger}$ Lin Xiong, ${ }^{\ddagger}$ Zhong-Wei Lu,${ }^{\dagger}$ Bo-Yu Zhen,${ }^{\dagger}$ Xin Sui,$\star$ Xue-Bin Deng, ${ }^{\dagger}$ Ling Chen, ${ }^{\dagger} * *$ and Li-Ming $\mathrm{Wu}^{\dagger} * *$

'Beijing Key Laboratory of Energy Conversion and Storage Materials, College of Chemistry, Beijing Normal University, Beijing 100875, P. R. China

$\$$ Key Laboratory of Theoretical and Computational Photochemistry, Ministry of Education, Beijing Normal University, Beijing 100875, P. R. China 


\section{Experiment Section}

\section{Synthesis of $\left(\mathrm{NH}_{4}\right)_{2} \mathrm{PO}_{3} \mathrm{~F}$}

A total of $1.4621 \mathrm{~g}$ of $\mathrm{H}_{3} \mathrm{PO}_{4}, 0.8960 \mathrm{~g}$ of $\mathrm{CO}\left(\mathrm{NH}_{2}\right)_{2}$, and $0.4255 \mathrm{~g}$ of $\mathrm{NH}_{4} \mathrm{HF}_{2}$ in a molar ratio of 2:2:1 weighted in glovebox were mixed in a polytetrafluoroethylene reaction vessel. The vessel was capped and heated over $45 \mathrm{~min}$ to $150^{\circ} \mathrm{C}$, held for $200 \mathrm{~min}$, and then cooled to $30^{\circ} \mathrm{C}$ at a rate of $0.04{ }^{\circ} \mathrm{C} / \mathrm{min}$. Finally, white product was obtained with a high yield of $97.8 \%$.

\section{Tables and Figures}

Table S1. The K/P ratio comparison between the loading ratio, single crystal diffraction data refinement and the ICP data of the produced crystals.

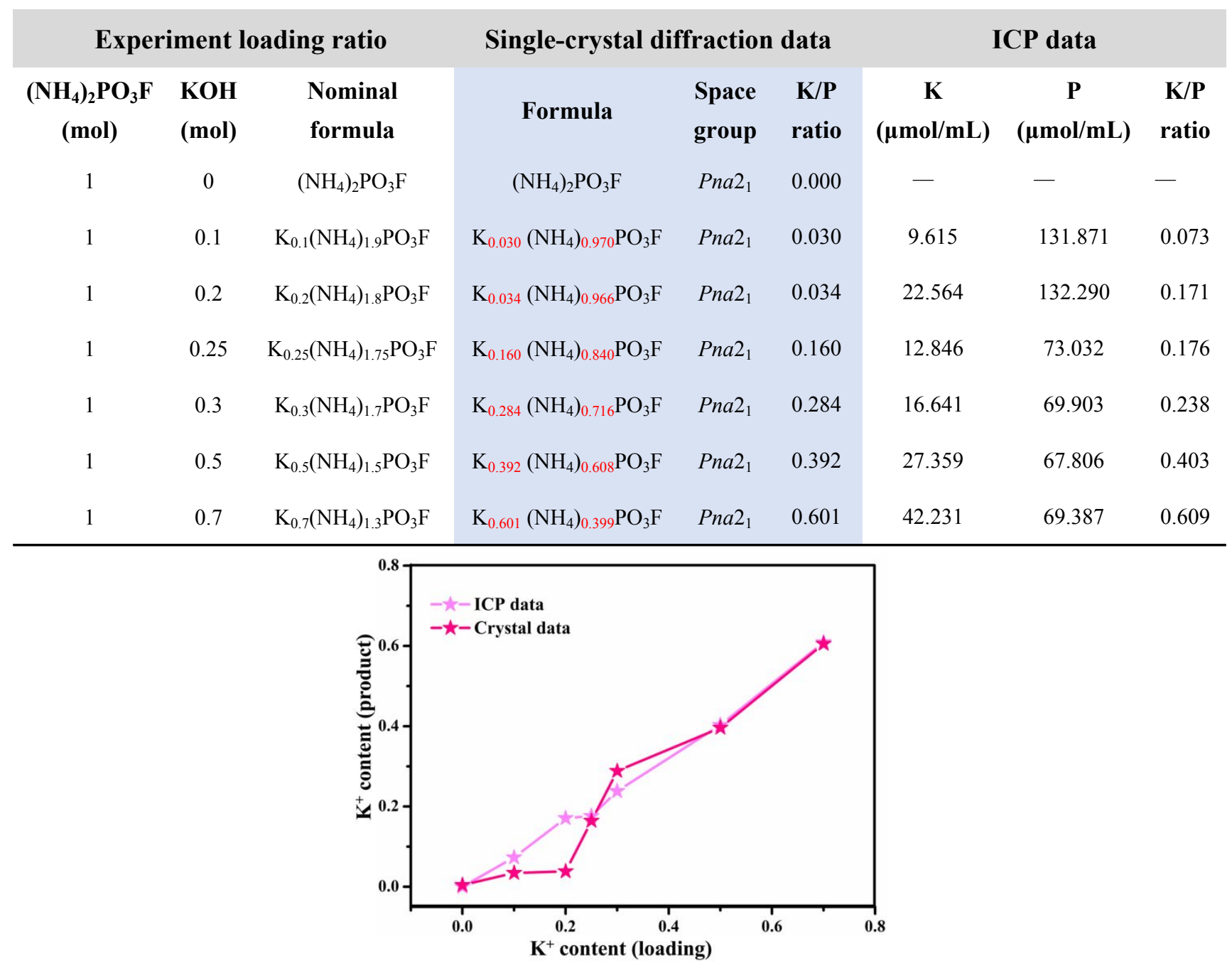

Figure S1. The $\mathrm{K}^{+}$content comparison between the loading ratio, single crystal diffraction and ICP data of the as-synthesized product. 
Table S2. Atomic coordinates and equivalent isotropic displacement parameters for $\left(\mathrm{NH}_{4}\right)_{2} \mathrm{PO}_{3} \mathrm{~F}$ and partial atomic occupancy for $\mathrm{K}_{0.1}\left(\mathrm{NH}_{4}\right)_{1.9} \mathrm{PO}_{3} \mathrm{~F}, \mathrm{~K}_{0.2}\left(\mathrm{NH}_{4}\right)_{1.8} \mathrm{PO}_{3} \mathrm{~F}, \mathrm{~K}_{0.25}\left(\mathrm{NH}_{4}\right)_{1.75} \mathrm{PO}_{3} \mathrm{~F}, \mathrm{~K}_{0.3}\left(\mathrm{NH}_{4}\right)_{1.7} \mathrm{PO}_{3} \mathrm{~F}, \mathrm{~K}_{0.5}\left(\mathrm{NH}_{4}\right)_{1.5} \mathrm{PO}_{3} \mathrm{~F}$, and $\mathrm{K}_{0.7}\left(\mathrm{NH}_{4}\right)_{1.3} \mathrm{PO}_{3} \mathrm{~F}^{*}$ *

\begin{tabular}{|c|c|c|c|c|c|c|c|}
\hline \multirow[b]{2}{*}{ Atom } & \multirow[b]{2}{*}{ Ox. } & \multicolumn{3}{|c|}{$\left(\mathrm{NH}_{4}\right)_{2} \mathrm{PO}_{3} \mathrm{~F}$} & \multicolumn{2}{|c|}{ HTP (300 K) } & \multirow[b]{2}{*}{$U_{\text {eq }}\left(\AA^{2}\right)$} \\
\hline & & Wyck. & Site Occ. & $x / a$ & $y / b$ & $z / c$ & \\
\hline $\mathrm{P}$ & +5 & $4 a$ & 1 & $0.1898(1)$ & $0.1260(1)$ & $0.0844(1)$ & $0.019(1)$ \\
\hline $\mathrm{O} 1$ & -2 & $4 a$ & 1 & $0.2865(2)$ & $0.0178(2)$ & $0.1558(3)$ & $0.029(1)$ \\
\hline $\mathrm{O} 2$ & -2 & $4 a$ & 1 & $0.1956(2)$ & $0.2271(2)$ & $0.2422(4)$ & $0.038(1)$ \\
\hline $\mathrm{O} 3$ & -2 & $4 a$ & 1 & $0.2183(2)$ & $0.1554(2)$ & $-0.1557(3)$ & $0.031(1)$ \\
\hline $\mathrm{F}$ & -1 & $4 a$ & 1 & 0 & $0.0822(1)$ & $0.0908(4)$ & $0.040(1)$ \\
\hline $\mathrm{N} 1$ & -3 & $4 a$ & 1 & $0.6412(2)$ & $0.0401(2)$ & $0.1012(5)$ & $0.027(1)$ \\
\hline H1 & +1 & $4 a$ & 1 & $0.5308(2)$ & $0.0314(2)$ & $0.1090(4)$ & $0.021(6)$ \\
\hline $\mathrm{H} 2$ & +1 & $4 a$ & 1 & $0.6830(3)$ & $-0.0083(2)$ & $0.1930(4)$ & $0.025(8)$ \\
\hline $\mathrm{H} 3$ & +1 & $4 a$ & 1 & $0.6650(3)$ & $0.1097(2)$ & $0.1400(4)$ & $0.036(9)$ \\
\hline $\mathrm{H} 4$ & +1 & $4 a$ & 1 & $0.6690(3)$ & $0.0250(2)$ & $-0.0280(3)$ & $0.036(1)$ \\
\hline $\mathrm{N} 2$ & -3 & $4 a$ & 1 & $0.0898(2)$ & $-0.1918(2)$ & $0.0941(6)$ & $0.032(1)$ \\
\hline H5 & +1 & $4 a$ & 1 & $-0.0020(2)$ & $-0.1860(2)$ & $0.1540(4)$ & $0.053(1)$ \\
\hline H6 & +1 & $4 a$ & 1 & $0.1410(3)$ & $-0.1283(2)$ & $0.0820(6)$ & $0.037(7)$ \\
\hline $\mathrm{H} 7$ & +1 & $4 a$ & 1 & $0.1510(3)$ & $-0.2350(2)$ & $0.1840(4)$ & $0.038(9)$ \\
\hline $\mathrm{H} 8$ & +1 & $4 a$ & 1 & $0.0850(4)$ & $-0.2270(3)$ & $-0.0240(4)$ & $0.061(1)$ \\
\hline \multicolumn{8}{|c|}{$\mathrm{K}_{0.1}\left(\mathrm{NH}_{4}\right)_{1.9} \mathrm{PO}_{3} \mathrm{~F}^{*(1)}$} \\
\hline N2 & -3 & $4 a$ & 0.970 & $0.4138(4)$ & $0.1915(2)$ & $1.4377(9)$ & $0.027(1)$ \\
\hline K & +1 & $4 a$ & 0.030 & $0.4138(4)$ & $0.1915(2)$ & $1.4377(9)$ & $0.027(1)$ \\
\hline \multicolumn{8}{|c|}{$\mathrm{K}_{0.2}\left(\mathrm{NH}_{4}\right)_{1.8} \mathrm{PO}_{3} \mathrm{~F}^{*(1)}$} \\
\hline N2 & -3 & $4 a$ & 0.966 & $0.0853(3)$ & $-0.1914(2)$ & $0.9156(7)$ & $0.024(1)$ \\
\hline
\end{tabular}


Supporting Information

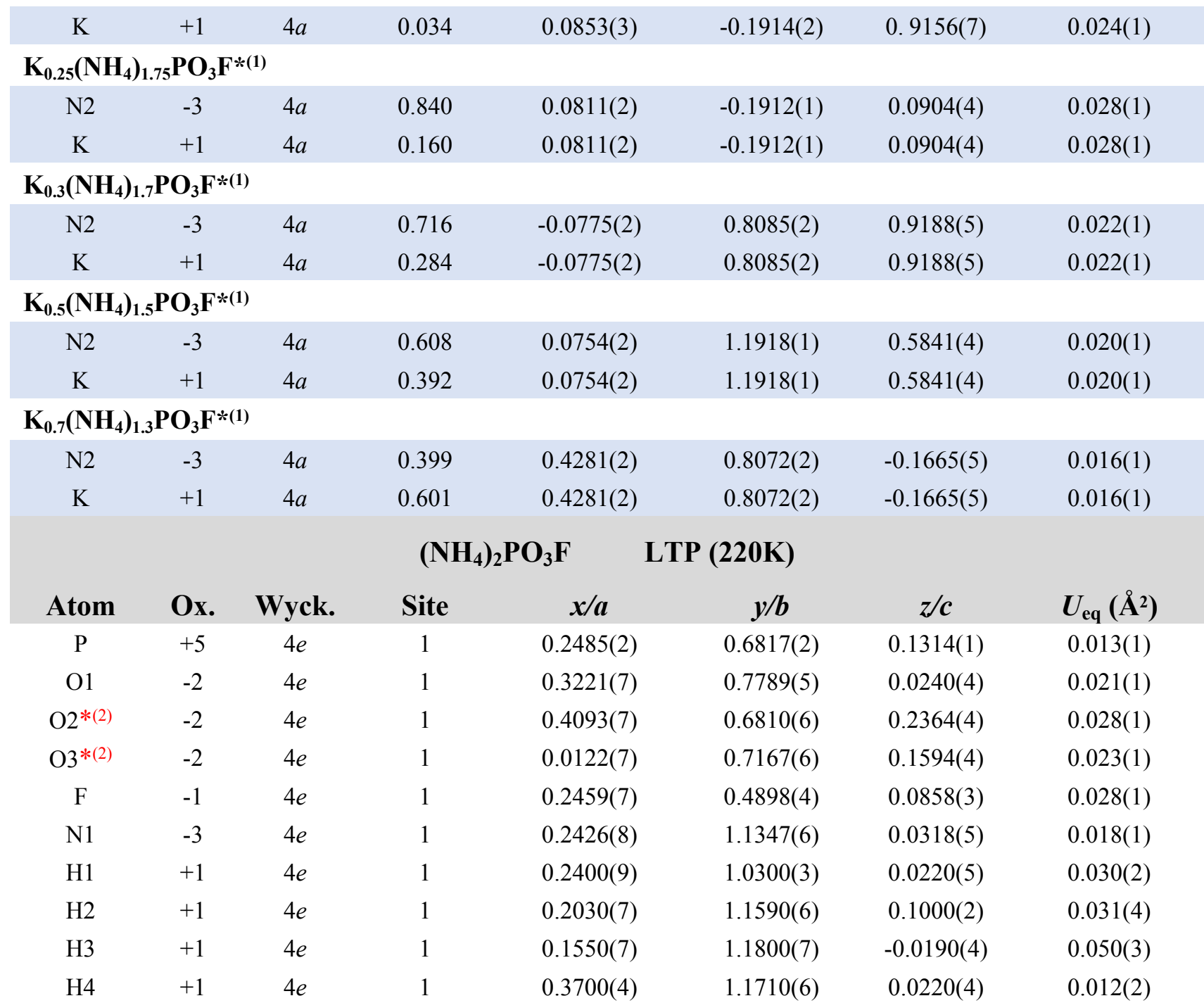




\begin{tabular}{llllllll}
$\mathrm{N} 2$ & -3 & $4 e$ & 1 & $0.2344(9)$ & $0.5900(7)$ & $-0.1894(5)$ & $0.021(1)$ \\
$\mathrm{H} 5$ & +1 & $4 e$ & 1 & $0.1840(9)$ & $0.4930(4)$ & $-0.1830(5)$ & $0.050(3)$ \\
$\mathrm{H} 6$ & +1 & $4 e$ & 1 & $0.2660(9)$ & $0.6310(7)$ & $-0.1220(3)$ & $0.050(3)$ \\
$\mathrm{H} 7$ & +1 & $4 e$ & 1 & $0.1410(7)$ & $0.6530(6)$ & $-0.2240(4)$ & $0.030(2)$ \\
$\mathrm{H} 8$ & +1 & $4 e$ & 1 & $0.3490(6)$ & $0.5880(8)$ & $-0.2290(4)$ & $0.040(2)$ \\
\hline
\end{tabular}

*: (1) nominal formula where $x$ represents the loading ratio of $\mathrm{K}^{+}$.

(2) For the sake of clarity and simplicity, the O2, O3 in Table S2 is marked as O3, O2 in Figure 2, respectively. 
Table S3-1. The crystal structure of $\left(\mathrm{NH}_{4}\right)_{2} \mathrm{PO}_{3} \mathrm{~F}$ collected in different temperatures.*

\begin{tabular}{|c|c|c|c|c|c|c|c|}
\hline \multirow{2}{*}{$\begin{array}{ll} & \left(\mathbf{N H}_{4}\right)_{2} \mathbf{P O}_{3} \mathbf{F} \\
\mathrm{T}(\mathrm{K}) & \end{array}$} & \multicolumn{4}{|c|}{ RTP } & \multicolumn{3}{|c|}{ LTP } \\
\hline & $300 \mathrm{~K}$ & $280 \mathrm{~K}$ & $260 \mathrm{~K}$ & $252 \mathrm{~K}$ & $238 \mathrm{~K}$ & $230 \mathrm{~K}$ & $220 \mathrm{~K}$ \\
\hline Formula weight & 134.05 & 67.03 & 134.05 & 134.05 & 134.05 & 134.05 & 107.24 \\
\hline Crystal system & \multicolumn{4}{|c|}{ Orthorhombic } & \multicolumn{3}{|c|}{ Monoclinic } \\
\hline Crystal color & colorless & colorless & colorless & colorless & colorless & colorless & colorless \\
\hline Space group & \multicolumn{4}{|c|}{$\begin{array}{l}\text { Pna2(1) } \\
\text { (NO. 33) }\end{array}$} & \multicolumn{3}{|c|}{$\begin{array}{l}P 2(1) / n \\
(\mathrm{NO} .14)\end{array}$} \\
\hline$a(\AA)$ & $7.9411(3)$ & $7.9344(3)$ & $7.9296(3)$ & $7.9292(2)$ & $6.0598(4)$ & $6.0581(4)$ & $6.0577(4)$ \\
\hline$b(\AA)$ & $11.3394(5)$ & $11.3381(4)$ & $11.3383(4)$ & $11.3401(4)$ & $7.8788(5)$ & $7.8735(5)$ & $7.8714(5)$ \\
\hline$c(\AA)$ & $6.0330(3)$ & $6.0281(2)$ & $6.0243(2)$ & $6.0235(2)$ & $11.2274(7)$ & $11.2215(7)$ & $11.2223(7)$ \\
\hline$\alpha=\gamma($ deg. $)$ & 90 & 90 & 90 & 90 & 90 & 90 & 90 \\
\hline$\beta$ (deg.) & 90 & 90 & 90 & 90 & 92.234 & 92.254 & 92.255 \\
\hline$V\left(\AA^{3}\right)$ & $543.26(4)$ & $542.29(3)$ & $541.63(4)$ & $541.62(3)$ & $535.63(6)$ & $534.83(6)$ & $534.69(6)$ \\
\hline$Z$ & 4 & 8 & 4 & 4 & 4 & 4 & 5 \\
\hline $\mathrm{D}_{\mathrm{c}}\left(\mathrm{g} \cdot \mathrm{cm}^{-3}\right)$ & 1.639 & 1.642 & 1.644 & 1.644 & 1.662 & 1.665 & 1.665 \\
\hline$\mu\left(\mathrm{mm}^{-1}\right)$ & 0.444 & 0.445 & 0.445 & 0.445 & 0.450 & 0.451 & 0.451 \\
\hline $\mathrm{F}(000)$ & 280 & 280 & 280 & 280 & 280 & 280 & 280 \\
\hline GOOF on $F^{2}$ & 1.210 & 1.140 & 1.002 & 1.089 & 1.257 & 1.249 & 1.289 \\
\hline$R_{1}, w R_{2}(I>2 \sigma(I))^{b}$ & $0.0428,0.0950$ & $0.0418,0.0896$ & $0.0351,0.0839$ & $0.0321,0.0713$ & $0.0953,0.2080$ & $0.0886,0.1993$ & $0.0970,0.2070$ \\
\hline$R_{1}, w R_{2}$ (all data $)^{b}$ & $0.0507,0.0999$ & $0.0525,0.0950$ & $0.0459,0.0904$ & $0.0409,0.0760$ & $0.1061,0.2135$ & $0.1006,0.2050$ & $0.1077,0.2120$ \\
\hline Flack parameter & 0.00 & 0.00 & 0.00 & 0.00 & none & none & none \\
\hline Largest diff peak/hole, e $\cdot \AA^{-3}$ & $0.310 /-0.550$ & $0.313 /-0.451$ & $0.302 /-0.252$ & $0.314 /-0.216$ & $1.369 /-0.538$ & $1.261 /-0.648$ & $1.272 /-0.573$ \\
\hline
\end{tabular}

* Special attention is paid when the temperature is closing to the $T_{c}$, the mounted crystal is kept on the goniometer still for at least about 40 min. to ensure the equilibrium of the phase transition process before the low temperature diffraction data are collected. 
Table S3-2. The crystal structure of $\mathrm{K}_{0.1}\left(\mathrm{NH}_{4}\right)_{1.9} \mathrm{PO}_{3} \mathrm{~F}$ collected in different temperatures.

\begin{tabular}{|c|c|c|c|c|c|c|c|c|}
\hline \multirow{2}{*}{$\begin{array}{l}\mathbf{K}_{\mathbf{0 . 1}}\left(\mathrm{NH}_{4}\right)_{1.9} \mathbf{P O}_{3} \mathbf{F} \\
\mathrm{T}(\mathrm{K})\end{array}$} & \multicolumn{3}{|c|}{ RTP } & \multicolumn{5}{|c|}{ LTP } \\
\hline & $300 \mathrm{~K}$ & $280 \mathrm{~K}$ & $260 \mathrm{~K}$ & $240 \mathrm{~K}$ & $220 \mathrm{~K}$ & $200 \mathrm{~K}$ & $180 \mathrm{~K}$ & $160 \mathrm{~K}$ \\
\hline Formula weight & 120.09 & 107.49 & 134.74 & 134.12 & 134.37 & 134.87 & 134.81 & 134.93 \\
\hline Crystal system & & Orthorhombic & & & & Monoclinic & & \\
\hline Crystal color & colorless & colorless & colorless & colorless & colorless & colorless & colorless & colorless \\
\hline Space group & & $\begin{array}{l}\text { Pna2(1) } \\
\text { (NO. 33) }\end{array}$ & & & & $\begin{array}{l}P 2(1) / n \\
(\mathrm{NO} .14)\end{array}$ & & \\
\hline$a(\AA)$ & 7.9395 & 7.9325 & 7.9299 & 6.0443 & 6.0447 & 6.0454 & 6.0442 & 6.0430 \\
\hline$b(\AA)$ & 11.3139 & 11.3130 & 11.3151 & 7.8705 & 7.8661 & 7.8617 & 7.8558 & 7.8506 \\
\hline$c(\AA)$ & 6.0289 & 6.0242 & 6.0217 & 11.1958 & 11.1926 & 11.1890 & 11.1835 & 11.1780 \\
\hline$\alpha=\gamma($ deg. $)$ & 90 & 90 & 90 & 90 & 90 & 90 & 90 & 90 \\
\hline$\beta$ (deg.) & 90 & 90 & 90 & 92.271 & 92.297 & 92.319 & 92.340 & 92.373 \\
\hline$V\left(\AA^{3}\right)$ & $541.56(2)$ & $540.61(3)$ & $540.31(3)$ & $532.18(4)$ & $531.76(4)$ & $531.35(3)$ & $530.57(3)$ & $529.84(3)$ \\
\hline$Z$ & 4 & 5 & 4 & 4 & 4 & 4 & 4 & 4 \\
\hline $\mathrm{D}_{\mathrm{c}}\left(\mathrm{g} \cdot \mathrm{cm}^{-3}\right)$ & 1.473 & 1.651 & 1.656 & 1.674 & 1.678 & 1.686 & 1.688 & 1.692 \\
\hline$\mu\left(\mathrm{mm}^{-1}\right)$ & 1.155 & 0.456 & 0.468 & 0.455 & 0.463 & 0.479 & 0.478 & 0.483 \\
\hline$F(000)$ & 236 & 281 & 281 & 280 & 281 & 282 & 281 & 282 \\
\hline GOOF on $F^{2}$ & 0.990 & 1.124 & 1.1132 & 1.605 & 1.252 & 1.570 & 1.466 & 1.424 \\
\hline$R_{1}, w R_{2}(I>2 \sigma(I))^{b}$ & $0.0500,0.1312$ & $0.0349,0.0825$ & $0.0412,0.1040$ & $0.0779,0.1936$ & $0.0663,0.1378$ & $0.0731,0.1880$ & $0.0677,0.1748$ & $0.0640,0.1686$ \\
\hline$R_{1}, w R_{2}$ (all data $)^{b}$ & $0.0555,0.1351$ & $0.0391,0.0843$ & $0.0449,0.1059$ & $0.0852,0.1980$ & $0.0770,0.1416$ & $0.0781,0.1903$ & $0.0751,0.1785$ & $0.0688,0.1707$ \\
\hline Flack parameter & 0.23 & 0.00 & 0.00 & none & none & none & none & none \\
\hline Largest diff peak/hole, e $\cdot \AA^{-3}$ & $0.463 /-0.483$ & $0.314 /-0.274$ & $0.421 /-0.460$ & $0.987 /-1.003$ & $0.753 /-0.492$ & $0.837 /-1.200$ & $0.825 /-1.182$ & $0.872 /-1.084$ \\
\hline
\end{tabular}


Table S3-3. The crystal structure of $\mathrm{K}_{0.2}\left(\mathrm{NH}_{4}\right)_{1.8} \mathrm{PO}_{3} \mathrm{~F}$ collected in different temperatures.

\begin{tabular}{|c|c|c|c|c|c|c|c|c|c|}
\hline \multirow{2}{*}{$\begin{array}{l}\mathbf{K}_{\mathbf{0 . 2}}\left(\mathrm{NH}_{4}\right)_{1.8} \mathbf{P O}_{3} \mathbf{F} \\
\mathrm{T}(\mathrm{K})\end{array}$} & \multicolumn{4}{|c|}{ RTP } & \multicolumn{5}{|c|}{ LTP } \\
\hline & $306 \mathrm{~K}$ & $280 \mathrm{~K}$ & $260 \mathrm{~K}$ & $240 \mathrm{~K}$ & $230 \mathrm{~K}$ & $220 \mathrm{~K}$ & $210 \mathrm{~K}$ & $200 \mathrm{~K}$ & $190 \mathrm{~K}$ \\
\hline Formula weight & 135.06 & 134.18 & 108.00 & 134.62 & 135.12 & 135.56 & 135.43 & 135.25 & 135.62 \\
\hline Crystal system & \multicolumn{4}{|c|}{ Orthorhombic } & \multicolumn{5}{|c|}{ Monoclinic } \\
\hline Crystal color & colorless & colorless & colorless & colorless & colorless & colorless & colorless & colorless & colorless \\
\hline Space group & \multicolumn{4}{|c|}{$\begin{array}{l}\text { Pna2(1) } \\
\text { (NO. 33) }\end{array}$} & \multicolumn{5}{|c|}{$\begin{array}{l}P 2(1) / n \\
(\mathrm{NO} .14)\end{array}$} \\
\hline$a(\AA)$ & $7.9368(4)$ & $7.9319(3)$ & $7.9299(3)$ & $7.9285(3)$ & $6.0410(2)$ & $6.0404(2)$ & $6.0398(2)$ & $6.0398(2)$ & $6.0395(2)$ \\
\hline$b(\AA)$ & $11.2965(6)$ & $11.2950(4)$ & $11.2968(4)$ & $11.3009(4)$ & $7.8740(3)$ & $7.8708(2)$ & $7.8673(3)$ & $7.8659(3)$ & $7.8631(3)$ \\
\hline$c(\AA)$ & $6.0229(3)$ & $6.0190(2)$ & $6.0169(2)$ & $6.0152(2)$ & $11.1841(4)$ & 11.1807(3) & $11.1779(4)$ & $11.1767(4)$ & $11.1748(5)$ \\
\hline$\alpha=\gamma($ deg. $)$ & 90 & 90 & 90 & 90 & 90 & 90 & 90 & 90 & 90 \\
\hline$\beta$ (deg.) & 90 & 90 & 90 & 90 & 92.260 & 92.268 & 92.279 & 92.289 & 92.309 \\
\hline$V\left(\AA^{3}\right)$ & $540.0(0)$ & $539.25(3)$ & $539.01(3)$ & $538.96(3)$ & $531.58(3)$ & $531.15(3)$ & $530.72(3)$ & $530.56(3)$ & $530.25(4)$ \\
\hline $\mathrm{D}_{\mathrm{c}}\left(\mathrm{g} \cdot \mathrm{cm}^{-3}\right)$ & 1.661 & 1.653 & 1.664 & 1.659 & 1.688 & 1.695 & 1.695 & 1.693 & 1.699 \\
\hline$\mu\left(\mathrm{mm}^{-1}\right)$ & 0.477 & 0.451 & 0.476 & 0.465 & 0.487 & 0.501 & 0.497 & 0.492 & 0.504 \\
\hline $\mathrm{F}(000)$ & 282 & 280 & 282 & 281 & 282 & 283 & 283 & 282 & 283 \\
\hline GOOF on $F^{2}$ & 1.157 & 1.162 & 0.975 & 0.938 & 1.216 & 1.164 & 1.088 & 1.189 & 1.187 \\
\hline$R_{1}, w R_{2}(I>2 \sigma(I))^{b}$ & $0.0405,0.0941$ & $0.0414,0.0925$ & $0.0416,0.1119$ & $0.0396,0.1074$ & $0.0500,0.1422$ & $0.0396,0.0896$ & $0.0426, \quad 0.1234$ & $0.0480,0.1049$ & $0.0455,0.1008$ \\
\hline$R_{1}, w R_{2}(\text { all data })^{b}$ & $0.0442,0.0957$ & $0.0445,0.0938$ & $0.0462,0.1172$ & $0.0443,0.1123$ & $0.0551,0.1464$ & $0.0445,0.0920$ & $0.0487,0.1292$ & $0.0516,0.1063$ & $0.0493,0.1026$ \\
\hline Flack parameter & 0.00 & 0.14 & 0.00 & 0.00 & none & none & none & none & none \\
\hline Largest diff peak/hole, e. $\AA^{-3}$ & $0.362 /-0.338$ & $0.354 /-0.319$ & $0.311 /-0.611$ & $0.345 /-0.635$ & $0.526 /-0.960$ & $0.346 /-0.344$ & $0.494 /-0.791$ & $0.435 /-0.417$ & $0.416 /-0.447$ \\
\hline
\end{tabular}


Table S3-4. The crystal structure of $\mathrm{K}_{0.3}\left(\mathrm{NH}_{4}\right)_{1.7} \mathrm{PO}_{3} \mathrm{~F}$ collected in different temperatures.

\begin{tabular}{|c|c|c|c|c|c|c|c|c|c|}
\hline \multirow{2}{*}{$\begin{array}{l}\mathbf{K}_{\mathbf{0 . 3}}\left(\mathrm{NH}_{\mathbf{4}}\right)_{\mathbf{1 . 7}} \mathbf{P O}_{\mathbf{3}} \mathbf{F} \\
\mathrm{T}(\mathrm{K})\end{array}$} & \multicolumn{6}{|c|}{ RTP } & \multicolumn{3}{|c|}{ LTP } \\
\hline & $304 \mathrm{~K}$ & $260 \mathrm{~K}$ & $220 \mathrm{~K}$ & $190 \mathrm{~K}$ & $170 \mathrm{~K}$ & $140 \mathrm{~K}$ & $120 \mathrm{~K}$ & $110 \mathrm{~K}$ & $90 \mathrm{~K}$ \\
\hline Formula weight & 141.33 & 141.14 & 141.46 & 141.08 & 139.90 & 140.06 & 140.89 & 140.89 & 140.89 \\
\hline Crystal system & \multicolumn{6}{|c|}{ Orthorhombic } & \multicolumn{3}{|c|}{ Monoclinic } \\
\hline Crystal color & colorless & colorless & colorless & colorless & colorless & colorless & colorless & colorless & colorless \\
\hline Space group & \multicolumn{6}{|c|}{$\begin{array}{c}\text { Pna }_{1} \\
\text { (NO. 33) }\end{array}$} & \multicolumn{3}{|c|}{$\begin{array}{l}P 2(1) / n \\
(\mathrm{NO} .14)\end{array}$} \\
\hline$a(\AA)$ & $7.9297(1)$ & $7.9247(3)$ & $7.9171(3)$ & $7.9076(3)$ & $7.9057(3)$ & $7.9048(3)$ & $5.9813(2)$ & $5.9756(3)$ & $5.9761(3)$ \\
\hline$b(\AA)$ & $11.1111(2)$ & $11.1134(4)$ & $11.1090(5)$ & $11.0973(5)$ & $11.0964(5)$ & $11.0997(5)$ & $7.8672(2)$ & $7.8620(3)$ & $7.8611(3)$ \\
\hline$c(\AA)$ & $5.9820(1)$ & $5.9812(3)$ & $5.9766(3)$ & $5.9692(3)$ & $5.9676(3)$ & $5.9682(2)$ & $10.9965(4)$ & $10.9873(5)$ & $10.9870(5)$ \\
\hline$\alpha=\gamma($ deg. $)$ & 90 & 90 & 90 & 90 & 90 & 90 & 90 & 90 & 90 \\
\hline$\beta$ (deg.) & 90 & 90 & 90 & 90 & 90 & 90 & 91.916 & 91.939 & 91.941 \\
\hline$V\left(\AA^{3}\right)$ & $527.06(2)$ & $526.77(4)$ & $525.65(4)$ & $523.82(4)$ & $523.51(4)$ & $523.66(4)$ & $517.16(3)$ & $515.89(4)$ & $515.86(4)$ \\
\hline$Z$ & 4 & 4 & 4 & 4 & 4 & 4 & 4 & 4 & 4 \\
\hline $\mathrm{D}_{\mathrm{c}}\left(\mathrm{g} \cdot \mathrm{cm}^{-3}\right)$ & 1.781 & 1.780 & 1.787 & 1.789 & 1.775 & 1.777 & 1.810 & 1.814 & 1.814 \\
\hline$\mu\left(\mathrm{mm}^{-1}\right)$ & 0.685 & 0.679 & 0.690 & 0.681 & 0.679 & 0.685 & 0.684 & 0.685 & 0.685 \\
\hline $\mathrm{F}(000)$ & 294 & 294 & 294 & 293 & 289 & 289 & 293 & 293 & 293 \\
\hline GOOF on $F^{2}$ & 1.206 & 1.162 & 1.119 & 1.146 & 1.151 & 1.119 & 1.432 & 1.223 & 1.213 \\
\hline$R_{1}, w R_{2}(I>2 \sigma(I))^{b}$ & $0.0616,0.1558$ & $0.0594,0.1529$ & $0.0558,0.1457$ & $0.0622,0.1256$ & $0.0612,0.1123$ & $0.0604,0.1106$ & $0.0755,0.1886$ & $0.0801,0.1509$ & $0.0779,0.1429$ \\
\hline$R_{1}, w R_{2}$ (all data $)^{b}$ & $0.0720,0.1614$ & $0.0682,0.1571$ & $0.0696,0.1536$ & $0.0725,0.1298$ & 0.07330 .1166 & $0.0724,0.1148$ & $0.0908,0.1959$ & $0.0941,0.1564$ & $0.0904,0.1472$ \\
\hline Flack parameter & 0.00 & 0.00 & 0.00 & 0.23 & 0.10 & 0.10 & none & none & none \\
\hline Largest diff peak/hole, e $\cdot \AA^{-3}$ & $0.561 /-0.515$ & $0.551 /-0.467$ & $0.571 /-0.518$ & $0.573 /-0.596$ & $0.472 /-0.525$ & $0.476 /-0.627$ & $0.802 /-0.874$ & $0.657 /-0.694$ & $0.660 /-0.507$ \\
\hline
\end{tabular}


Table S3-5. The crystal structure of $\mathrm{K}_{0.5}\left(\mathrm{NH}_{4}\right)_{1.5} \mathrm{PO}_{3} \mathrm{~F}$ and $\mathrm{K}_{0.7}\left(\mathrm{NH}_{4}\right)_{1.3} \mathrm{PO}_{3} \mathrm{~F}$.

\begin{tabular}{|c|c|c|}
\hline & $\mathrm{K}_{0.5}\left(\mathrm{NH}_{4}\right)_{1.5} \mathrm{PO}_{3} \mathrm{~F}$ & $\mathrm{~K}_{0.7}\left(\mathrm{NH}_{4}\right)_{1.3} \mathrm{PO}_{3} \mathrm{~F}$ \\
\hline Formula weight & 142.22 & 148.43 \\
\hline Crystal system & \multicolumn{2}{|c|}{ Orthorhombic } \\
\hline Crystal color & colorless & colorless \\
\hline Space group & \multicolumn{2}{|c|}{ Pna $_{1}$} \\
\hline$a(\AA)$ & $7.9345(9)$ & $7.9303(4)$ \\
\hline$b(\AA)$ & $11.0298(1)$ & $10.8767(7)$ \\
\hline$c(\AA)$ & $5.9653(8)$ & $5.9330(3)$ \\
\hline$\alpha=\gamma($ deg. $)$ & 90 & 90 \\
\hline$\beta$ (deg.) & 90 & 90 \\
\hline$V\left(\AA^{3}\right)$ & $522.06(1)$ & $511.75(5)$ \\
\hline$Z$ & 4 & 4 \\
\hline $\mathrm{D}_{\mathrm{c}}\left(\mathrm{g} \cdot \mathrm{cm}^{-3}\right)$ & 1.809 & 1.926 \\
\hline$\mu\left(\mathrm{mm}^{-1}\right)$ & 0.767 & 1.020 \\
\hline $\mathrm{F}(000)$ & 292 & 302 \\
\hline GOOF on $F^{2}$ & 1.139 & 1.260 \\
\hline$R_{1}, w R_{2}(I>2 \sigma(I))^{b}$ & $0.0463,0.0979$ & $0.0768,0.1381$ \\
\hline$R_{1}, w R_{2}(\text { all data })^{b}$ & $0.0518,0.1002$ & $0.0843,0.1410$ \\
\hline Flack parameter & 0.13 & 0.03 \\
\hline Largest diff peak/hole & $0.439 /-0.489$ & $0.833 /-0.681$ \\
\hline
\end{tabular}

Table S4. The calculated exchange-correlation energy of $\mathrm{K}_{x}\left(\mathrm{NH}_{4}\right)_{2-x} \mathrm{PO}_{3} \mathrm{~F}$.

\begin{tabular}{|c|c|c|c|c|c|c|c|c|c|c|}
\hline $\mathrm{K}^{+}$content & \multicolumn{2}{|c|}{0.00} & \multicolumn{2}{|c|}{0.3} & \multicolumn{2}{|c|}{0.50} & \multicolumn{2}{|c|}{0.75} & \multicolumn{2}{|c|}{1.00} \\
\hline Phase & LTP & HTP & LTP & HTP & LTP & HTP & LTP & HTP & LTP & HTP \\
\hline$E_{x c}(e V)$ & -310.810 & -310.780 & -289.258 & -289.244 & -267.773 & -267.807 & -246.317 & -246.353 & -224.866 & -224.909 \\
\hline $\begin{array}{l}E_{x c}(H T P)- \\
E_{x c}(L T P) \\
(e V)\end{array}$ & \multicolumn{2}{|c|}{0.030} & \multicolumn{2}{|c|}{0.014} & \multicolumn{2}{|c|}{-0.035} & \multicolumn{2}{|c|}{-0.036} & \multicolumn{2}{|c|}{-0.043} \\
\hline
\end{tabular}


Table S5. The calculated details of entropy change $\Delta S$, enthalpy change $\Delta H$ and the ratio of possible orientations $N$ for $\mathrm{K}_{x}\left(\mathrm{NH}_{4}\right)_{2-x} \mathrm{PO}_{3} \mathrm{~F}(x=0,0.1,0.2)$ with the heat flow $80 \mathrm{~K} / \mathrm{min}$ in heating cycle and cooling cycle.

Calculation details:

$\left(\mathrm{NH}_{4}\right)_{2} \mathrm{PO}_{3} \mathrm{~F}$ in heating cycle for example.

Clausius inequation:

$$
\begin{aligned}
\Delta S & =\int_{T_{1}}^{\mathrm{T}_{2}} \frac{Q}{T} \mathrm{dT} \\
& \approx \frac{\Delta \mathrm{H}^{\exp }}{T_{c}} \\
& =\frac{4.2068 \mathrm{~J} \cdot \mathrm{g}^{-1} \times 134.047 \mathrm{~g} \cdot \mathrm{mol}^{-1}}{281.3 \mathrm{~K}} \\
& =2.005 \mathrm{~J} \cdot \mathrm{mol}^{-1} \cdot \mathrm{K}^{-1}
\end{aligned}
$$

Boltzmann equation:

$$
\begin{aligned}
& \begin{aligned}
\Delta S & =R \ln N \\
N & =\exp \left(\frac{\Delta S}{R}\right)=\exp \left(\frac{2.005 \mathrm{~J} \cdot \mathrm{mol}^{-1} \cdot \mathrm{K}^{-1}}{8.314 \mathrm{~J} \cdot \mathrm{mol}^{-1} \cdot \mathrm{K}^{-1}}\right) \quad(R: \text { the gas constant }) \\
& =1.273 \\
\Delta H^{\mathrm{cal}}=\mathrm{T} \Delta S & \\
& =281.31 \mathrm{~K} \times 2.005 \mathrm{~J} \cdot \mathrm{mol}^{-1} \cdot \mathrm{K}^{-1} \\
& =564.0266 \mathrm{~J} \cdot \mathrm{mol}^{-1}=4.2077 \mathrm{~J} / \mathrm{g}
\end{aligned}
\end{aligned}
$$

Table S6. Dipole moment for HTP and LTP of $\left(\mathrm{NH}_{4}\right)_{2} \mathrm{PO}_{3} \mathrm{~F}$ along $x, y, z$ directions.

\begin{tabular}{lcccccccc} 
Compound & & Anion & $\boldsymbol{x}$ & Total & $\boldsymbol{y}$ & Total & $\boldsymbol{z}$ & Total $\boldsymbol{z}$ \\
& & & -1.4980 & & 0.5940 & & 0.0180 & \\
& \multirow{2}{*}{$\mathrm{HTP}$} & $\left(\mathrm{PO}_{3} \mathrm{~F}\right)^{2-}$ & 1.4980 & & -0.5940 & & 0.0180 & \\
& & & -1.4980 & 0 & 0.5940 & 0 & 0.0180 & 0.0720 \\
$\left(\mathrm{NH}_{4}\right)_{2} \mathrm{PO}_{3} \mathrm{~F}$ & & & 1.4980 & & -0.5940 & & 0.0180 & \\
& & & -0.0109 & & 1.4580 & -0.3593 & \\
& \multirow{2}{*}{$\mathrm{LTP}$} & $\left(\mathrm{PO}_{3} \mathrm{~F}\right)^{2-}$ & 0.0109 & & 1.4580 & & 0.3593 & \multirow{2}{*}{0} \\
& & & -0.0109 & 0 & -1.4580 & 0 & -0.3593 & \\
& & & 0.0109 & & -1.4580 & & 0.3593 & \\
\hline
\end{tabular}


a

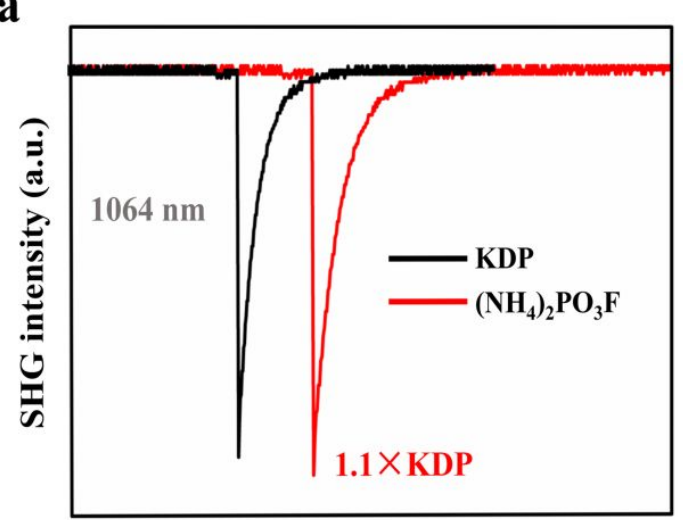

Times (ms)

c

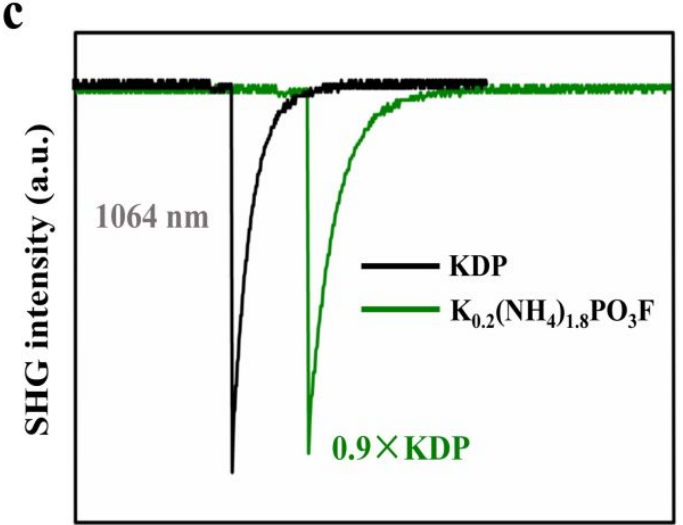

Times (ms) b

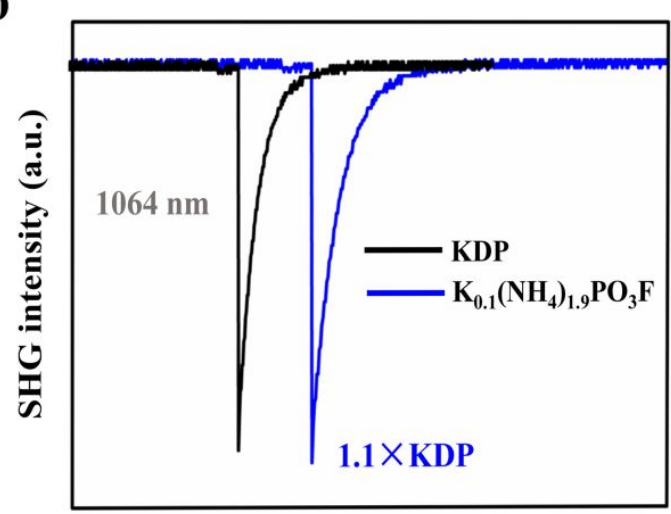

Times (ms)

d

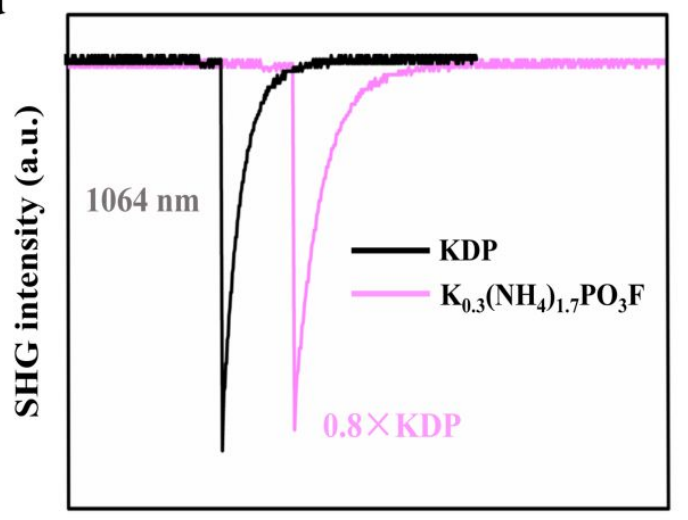

Times (ms)

Figure S2. The SHG effect comparison of HTP and KDP for $\mathrm{K}_{x}\left(\mathrm{NH}_{4}\right)_{2-x} \mathrm{PO}_{3} \mathrm{~F}(x=0$, $0.1,0.2,0.3)$ with the particle sizes of $150-212 \mu \mathrm{m}$ at $1064 \mathrm{~nm}$.

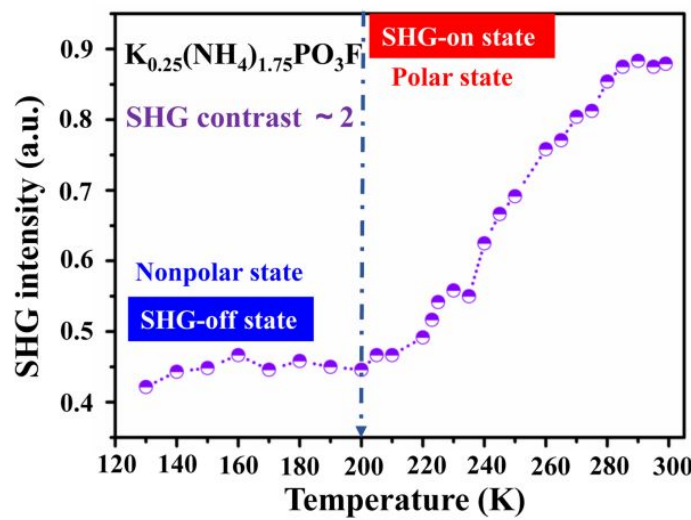

Figure S3. The temperature-dependent SHG for $\mathrm{K}_{0.25}\left(\mathrm{NH}_{4}\right)_{1.75} \mathrm{PO}_{3} \mathrm{~F}$. 